\title{
Paraplegia
}

\section{Psychological Factors in the Causation of Pressure Sores: Case Reports}

\author{
A. Heilporn \\ Centre de Traumatologie et de Readaption, Place A. Van Gehuchten 4, 1020 Brussels, \\ Belgium.
}

\section{Summary}

A high incidence of re-admissions to a spinal injuries unit by a small number of patients suffering from multiple pressure sores is described. The study shows an increased association of skin fragility and poor healing with an altered psychological behaviour. This combination of vulnerability to recurrent pressure sores in association with the pathological intellectual debility is described as 'ectodermic syndrome'. This restricts the eventual rehabilitative outcome.

Key words: Pressure sores; Psychological factors; Spinal cord injury; Paraplegia.

Most studies and articles dealing with pressure sores focus on tissue and metabolic factors, and most of the published data highlight the effects of physical pressure, anaemia and certain metabolic factors in the causation of pressure sores and their delay in healing.

Even though there are several publications on the psychological response to trauma amongst patients with spinal cord injury (SCI), there have been very few studies relating to its effect on the causation of pressure scores. ${ }^{1-4}$

We report on two case histories of patients who presented with multiple recurrent pressure sores and required several hospital admissions.

\section{Case history}

A man aged 36 years, a driving instructor, who had a car accident on 30 September 1985, and sustained multiple trauma with 48 hours of unconsciousness, a T4 complete paraplegia. He was discharged after initial rehabilitation, on 13 December 1986, when he was independent in all activities of daily living, and was able to return to professional activities. There were four hospitalisations from 1987 to February 1989, because of numerous pressure sores of variable severity and depth ( 2 ischial, 2 trochanteric, 2 anterior chest and heels). Surgical and nursing 
management included the use of a water bed. Slow healing occurred after 2 months. He required a permanent indwelling urethral catheter; there were no major urinary problems. Psychological evaluation revealed a behaviour of escape or flight, an unsolved problem of mourning and an initial immature personality; he refused psychotherapy.

\section{Case history}

A man aged 43 years, had an accident from a fall from a roof on 1 July 1975, sustaining a fracture of $\mathrm{L} 1$ and $\mathrm{L} 2$, with a partial paraplegia at $\mathrm{L} 1$ vertebral level. He was discharged home after rehabilitation, on 2 July 1976, but the rehabilitative outcome was poor and not commensurate with the level of lesion. He became dependent on his wife. There were eight hospital admissions between 1976 and 1988 all because of pressure sores; and another admission to a neurosurgical unit for pain with the application of epidural stimulation, but without success. Sacral and gluteal decubitus ulcers required about ten surgical procedures, including complex plastic surgery. Initial pressure sores healed in 2 months but subsequent pressure sores took over 7 months to heal. He required to spend a minimum of 3 months hospital stay for the treatment of each of these sores. There were no urinary complications. Psychological assessment revealed a previous history of depression and a further intellectual deterioration, episodes of confusion, and of anxiety attacks since the injury. There was no significant pyschological improvement despite psychiatric management.

\section{Discussion}

These two patients highlight the association of psychological factors with the occurrence of pressure sores. The patients presenting with what may be termed an 'ectodermic syndrome' which may be distinguished by the frequency and the length of hospitalisation. Even though the same patient is admitted several times the incidence of 'the ectodermic syndrome' is relatively small. Of the 716 patients with SCI admitted to the Centre de Traumatologie et de Readaptation in Brussels over a 10 year period (1979-1988) there were only 7 patients who could fit the syndrome described. This represents less than $1 \%$ of all admissions.

There is a denial of the disability often expressed in actions rather than in words in these patients. Inattention to pressure and bladder care are common manifestations of this. ${ }^{4}$ The inadequate behaviour often changes into a more positive attitude spontaneously thanks to rehabilitation. Patients with 'the ectodermic syndrome' mainly and often exclusively suffer from pressure sores. The lack of behavioural adjustment is thought to be due either to an impossibility to understand the necessity of care of a refusal to admit a new situation that has been imposed as a result of the disability. There has been no distinct evidence of selfdestruction in these patients. It is known that the criteria for psychological evaluation of patients with spinal cord injury is often difficult. ${ }^{4}$ The pre-morbid personality is certainly of great interest and importance in understanding the psychological evolution as a result of paralysis. ${ }^{5}$ In our study a feature of passivity or instability are mainly found in patients with 'the ectodermic syndrome'. In their rehabilitative evolution these patients are generally passive regarding the events of 
which they are the victim, and are rather lenient regarding the obligations of repeated and prolonged hospitalisation. Usually any suggestions of psychotherapy are refused and the problems are denied. Repeated instructions on the prevention of sores are either disregarded or forgotten. Pressure sore management presents major problems in these patients. The multiplicity and the recurrence of these lesions lead to fragile, wide and extensive scars on weight bearing parts of the body. The changes of the skin texture predisposes these patients to further recurrences. ${ }^{6}$ The slow healing seen in such patients appears to result from an alteration of the skin itself, thus the combination of repeated pressure sores and poor healing in the presence of psychological maladjustments presents with a general deterioration of function.

These patients are a particular burden to the spinal injury unit. The treatment of the organic lesion is complex and the psychological follow-up is deceiving, the social replacement is precarious, very often leading to a definitive placement in a care unit. This syndrome should be diagnosed as soon as possible in order to be realistic concerning their rehabilitation.

Returning these patients to a family environment should be avoided in this case and placement in a specialised medical unit if identified early will help to reduce the complications leading to an undeniable human and economic benefit.

\section{References}

1 Crenshaw RP, Vistnes LM. A decade of pressure sore research 1977-1987. Jour Rhabil Res Dev 1989: 26:63-74.

2 Cull HG, Smith OH. 'Preliminary note on demographic and personality correlated of decubitus ulcer incidence'. J Psychol 1973: 85:225-227.

3 Anderson TP, Andberg MM. 'Psychological factors associated with pressure sores. Arch Phys Med Rehabil 1979: 60:341-346.

4 Judd JK, Burrows GB. Liaison psychiatry in a spinal injuries unit. Paraplegia 1986: 24:6-19.

5 Judd JK, Burrows GB, Brown DJ. 'Depression following acute spinal cord injury'. Paraplegia 1986: 24:358-363.

6 Rodriguez GP, Claus Walker J. 'Biochemical changes in skin composition in spinal injury-A possible contribution to decubitus ulcers'. Paraplegia 1988: 26:302-309. 